\title{
El puente sobre el Tajo de Eiffel en España
}

\section{The bridge over the Tagus in Spain by Eiffel}

\author{
P. Plasencia-Lozano $^{(*)}$
}

\section{RESUMEN}

El texto describe y analiza el puente construido sobre el río Tajo por Gustave Eiffel en las cercanías de Cáceres, una de las obras más relevantes de la primera etapa del célebre ingeniero francés, y la mayor de las mismas construida en España. A partir de los planos hallados en diversos archivos, y de otras fuentes históricas, se analiza la obra y el proceso seguido para llevarla a cabo, y se repasa la importancia que tuvo tras su finalización. Al final del escrito, las conclusiones subrayan los puntos destacados del mismo y proponen distintas cuestiones relacionadas con las obras públicas históricas.

Palabras clave: Puente; Gustave Eiffel; Siglo XIX; Cáceres; Ferrocarril.

\section{ABSTRACT}

The text describes and analyses the bridge projected and constructed over the Tagus River in Spain by Gustave Eiffel, in the proximity of Cáceres. This is one of the most relevant works of the first stage of the notorious French engineer, and his biggest one built in Spain. After the analyses of the plans found in several archives, and of the other historic sources, the bridge and the construction process are described. Moreover, the importance of the structure after its construction is emphasized. Finally, the conclusions highlight the strong point of the article and suggest several questions concerning the historic public works.

Keywords: Bridge; Gustave Eiffel; XIX century; Cáceres; Railroads.

(*) Universidad de Oviedo (España).

Persona de contacto/Corresponding author: pedroplasencia@gmail.com (P. Plasencia-Lozano)

ORCID: http://orcid.org/oooo-0oo1-5240-0733 (P. Plasencia-Lozano)

Cómo citar este artículo/Citation: P. Plasencia-Lozano (2018). El puente sobre el Tajo de Eiffel en España. Informes de la Construcción, 70(551): e268. https://doi.org/10.3989/ic.60335

Copyright: (C) 2018 CSIC. Este es un artículo de acceso abierto distribuido bajo los términos de la licencia de uso y distribución Creative Commons Reconocimiento 4.o Internacional (CC BY 4.0). 


\section{INTRODUCCIÓN}

El puente construido sobre el río Tajo por Gustave Eiffel en España (fig. 1) se situó en la línea férrea que enlaza Madrid con Lisboa a través de Cáceres, unos $30 \mathrm{~km}$ al norte de esta última ciudad. Fue construido en 1881 y demolido hacia 1932.

Para la redacción del texto se han examinado documentos existentes en el Archivo Histórico Ferroviario de Madrid (AHF), el Archivo General de la Administración de Alcalá de Henares (AGA) y los Archives Nationales du monde du travail de Roubaix (ANMT). Además, se han consultado fuentes históricas y otros textos.

\section{LA LÍNEA MADRID-LISBOA EN EL SIGLO XIX}

El ferrocarril en España tuvo un desarrollo más lento que en otros países europeos: hasta 1850 apenas se habían puesto en servicio $56 \mathrm{~km}$ frente a los 2.500 de Francia, los 5.000 de Prusia o los 10.500 del Reino Unido ${ }^{1}$. Sin embargo, recibió un impulso notable en 1855 gracias a la promulgación de la Ley General de Caminos de Hierro.

Una de las líneas más relevantes a construir en la Península era la que uniría Madrid con Lisboa. A lo largo de los años se fueron encargando proyectos diversos englobados en dos corredores: el valle del Tajo y el del Guadiana. El primer trazado completo (1866) discurrió por este último, cruzando la frontera por Badajoz y Elvas, aunque con un recorrido de unos $870 \mathrm{~km}$ frente a los $504 \mathrm{~km}$ que hay en línea recta entre ambas ciudades. El trazado por el corredor del Tajo, significativamente más corto (unos $640 \mathrm{~km}$ ) fue completado en 1881.

\section{ESPACIO GEOGRÁFICO}

El punto más conflictivo de la parte española del trazado era el cruce del río Tajo. Durante años se especuló con la posibilidad de que dicho cruce se ubicara en las cercanías de Almaraz, redactándose incluso un proyecto (1), pero finalmente la alternativa elegida situó el cruce en el vado de Alconétar.

En esta zona se han construido destacados puentes a lo largo de la historia (2) (3), un fenómeno que encuentra explicación en la lógica adición de nuevas infraestructuras de transporte y en un hecho que modificó sustancialmente el espacio físico: la construcción de la presa de Alcántara en 1969. Situada unos $50 \mathrm{~km}$ aguas abajo de Alconétar, la presa aumentó la cota del agua, inundándose así los puentes existentes y obligando a construir algunos nuevos. Antes de 1969, para salvar el Tajo existían en Alconétar el puente romano (parcialmente arruinado), los restos del puente de Eiffel, el puente carretero de Cipriano Salvatierra (1927) y el puente ferroviario de Fernando del Pino (1933). Tras la construcción de la presa los restos del romano fueron trasladados a una nueva ubicación a mayor cota sobre el arroyo Guadancil, afluente del Tajo; además se construyeron nuevos puentes para dar continuidad al ferrocarril y la carretera (1967); recientemente se han construido dos arcos: el de la autovía A-66 (2006) y el de la nueva línea férrea (2018).

\section{EL PUENTE}

La construcción de líneas ferroviarias a finales del siglo XIX se desarrollaba mediante proyectos que abarcaban una enorme cantidad de kilómetros. Se definía el trazado, la disposición de estaciones o incluso el material móvil necesario para

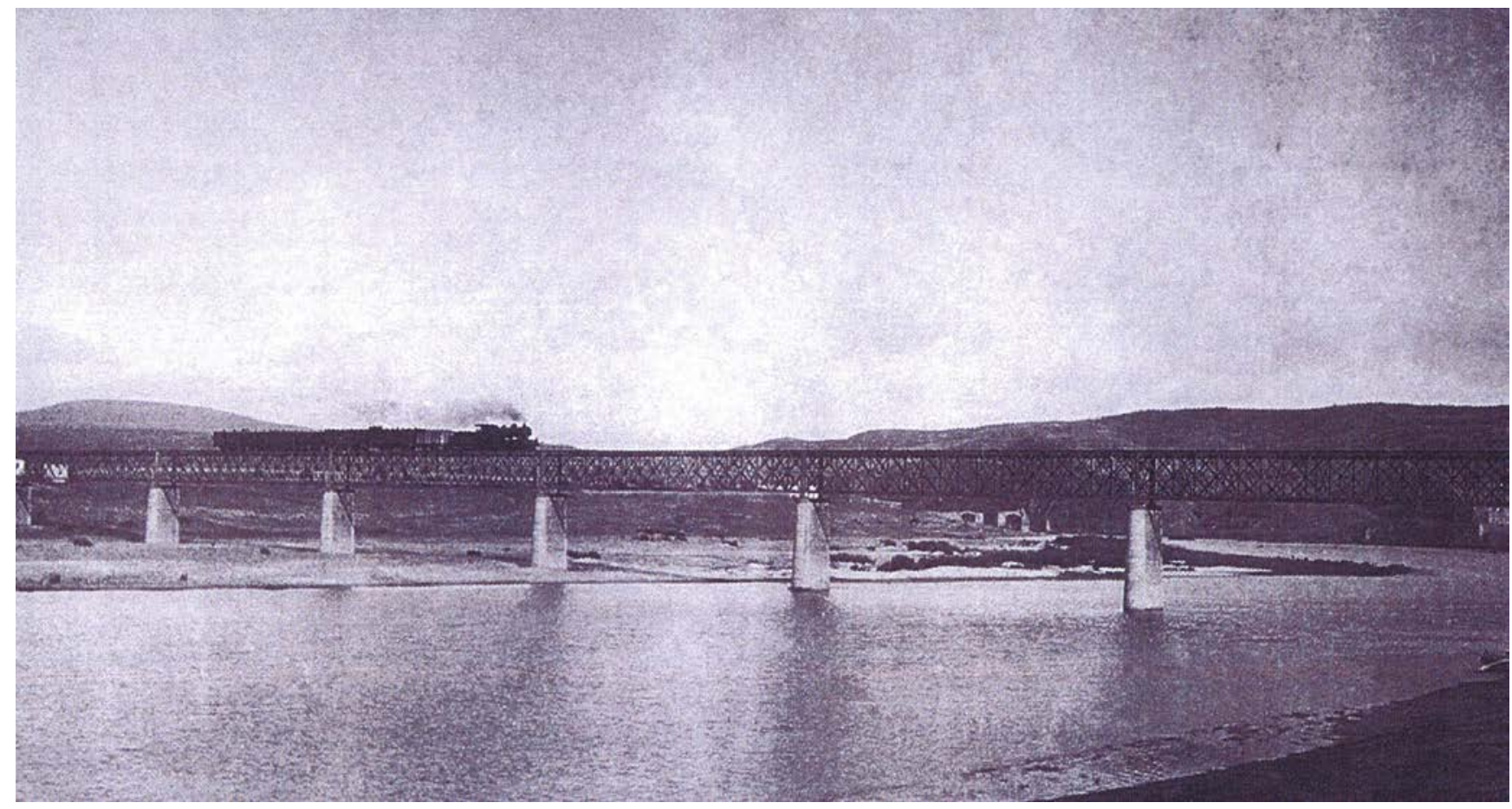

Figura 1. El puente del Tajo de Eiffel. Al fondo se observan los restos del puente romano de Alconétar (fotografía: J.M. Mellado).

\footnotetext{
${ }^{1}$ También pueden compararse las fechas de puesta en servicio de las primeras líneas: Reino Unido 1830, Prusia 1835, Bélgica 1835, Austria 1837, Holanda 1839, Italia 1839, Francia 1842, Polonia 1842, España peninsular 1848.
} 


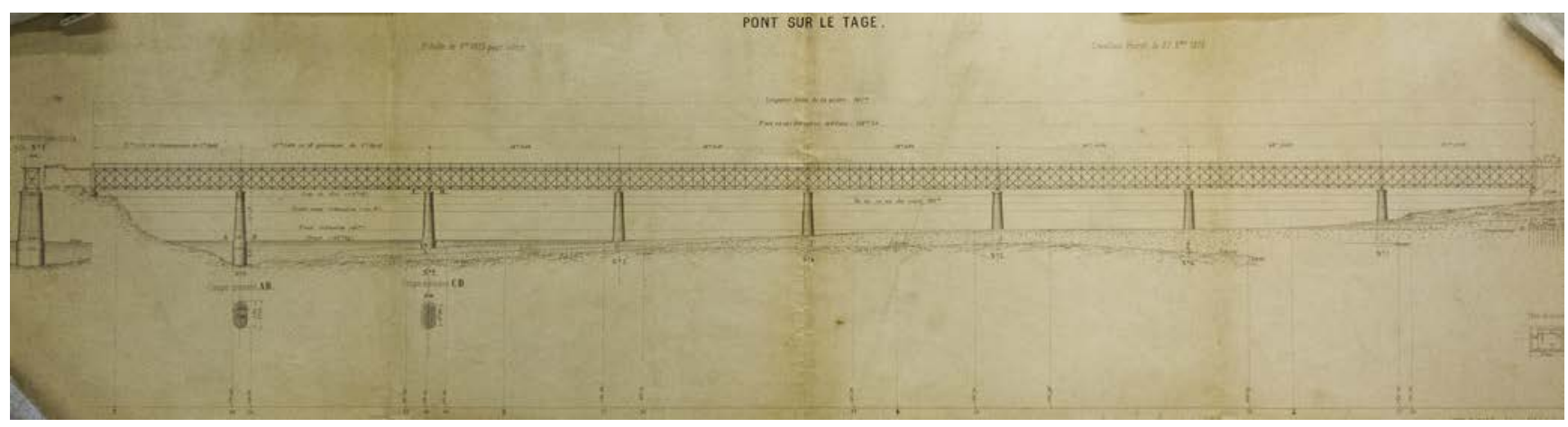

Figura 2. Alzado del puente (imagen: ANMT).

la posterior explotación (4). En cuanto a las estructuras, los proyectos de las líneas definían los puntos de paso principales y presupuestaban de un modo general las obras. Sin realizar cálculos estructurales, y a partir del conocimiento de la existencia de puentes ya construidos de un tipo u otro de celosía metálica ${ }^{2}$ con unas determinadas luces, se predefinían las estructuras principales a construir en la línea que se estaba proyectando, pero dejaban el detalle de las mismas a empresas especializadas, que desarrollaban posteriormente proyectos concretos. Así ocurrió por ejemplo con el puente del Guadiana en Mérida de Eduardo Peralta (5).

El puente del Tajo se situaba en la línea Malpartida de Plasencia-Cáceres, un tramo del itinerario Madrid-CáceresLisboa; dicha línea estaba promovida por la Sociedad de los ferrocarriles de Cáceres a Malpartida y a la frontera portuguesa (absorbida posteriormente por la Compañía de Madrid a Cáceres y Portugal). Pese a desconocer el proyecto general de la línea, sabemos que la estructura de cruce del río Tajo aparecía definida bajo algunos parámetros, como la ubicación exacta (PK 299+308, con Madrid como punto de origen), la longitud, la rasante o los materiales. El proyecto de línea recibió la aprobación del Ministerio de Fomento el 6 de julio de 1877 (6), que realizaba dos observaciones concernientes al puente del Tajo: que se aumentara en $1 \mathrm{~m}$ la cota de la estructura, y que en los apoyos se emplease el hierro forjado en lugar del fundido propuesto en un principio.

Tras la aprobación ministerial, la promotora de la línea encargó el proyecto de la estructura del Tajo a los Établissement Eiffel, quien también recibió el encargo de proyectar y construir el resto de estructuras metálicas de la línea (7).

\subsection{Descripción del puente}

Los planos conservados en el ANMT (8) permiten comprobar cómo el puente del Tajo era una estructura metálica roblonada de celosía continua de $367 \mathrm{~m}$, medida de extremo a extremo de viga (fig. 2). Estaba formado por 6 vanos centrales de 48,51 m y dos vanos extremos de 37,72 m entre ejes de apoyos, resultando una longitud total entre paramentos de estribos de $365 \mathrm{~m}$.

La estructura, de 5,40 m de altura y 5,50 de anchura, era de doble celosía tipo Warren con montantes espaciados. El puente era de piso superior, con viguetas y largueros de alma llena para vía única, y las vigas presentaban una separación de 3,50 m entre ejes. En la zona izquierda del puente se proyectó una pasarela adosada, de $75 \mathrm{~cm}$ de anchura con una barandilla de $1 \mathrm{~m}$ de altura, para facilitar las tareas de supervisión de estructura y para permitir el acceso o abandono del tren en caso de necesidad (fig. 3).

El perfil de los raíles era de tipo Vignole, separados $1.732 \mathrm{~mm}$, medidos de centro a centro. Deducimos de ello que el perfil dibujado medía $67 \mathrm{~mm}$, teniendo en cuenta que el ancho de la vía era de $1.668 \mathrm{~mm}$.

En relación a los estribos y pilas, eran de sillería y ladrillo (fig. 4). Los estribos alcanzaban una anchura de 4,80 m. Las pilas medían, a cota de estiaje, 6,30 x 2,80 m; estas medidas se reducían progresivamente conforme ascendían, y en la coronación presentaban unas dimensiones de 5,50 x 2,00 m.

En cuanto a la cimentación (fig. 6), el terreno donde se ubicaba el puente no presentaba una geología uniforme. Las pilas 1,2 y 6 fueron cimentadas sobre pizarra, y requirieron excavaciones de hasta $6,50 \mathrm{~m}$ bajo el nivel de estiaje. Para la ejecución fue diseñado un cajón de aire comprimido (fig. 5): una suerte de caparazón metálico de $2 \mathrm{~m}$ de altura cuyo interior, en el extremo inferior, presentaba las mismas dimensiones que la sección de la cimentación de las pilas. La superficie se encontraba cerrada con placas de 4 a $6 \mathrm{~mm}$ de espesor, quedando la base hueca; en la cara superior existía un agujero circular de 0,45 $\mathrm{m}$ de radio. La estructura se hallaba fuertemente armada, con angulares de hasta $80 x 80 x 8 \mathrm{~mm}$, y para facilitar su transporte fue dividida en 3 fragmentos, de aproximadamente $2,25 \mathrm{~m} \mathrm{x} 2 \mathrm{~m} \mathrm{x} \mathrm{3,20} \mathrm{m.}$

Las pilas 3, 4, 5 y 7 se dispusieron en un terreno con una capa de arena y grava que alcanzaba los 2,40 $\mathrm{m}$ de potencia en los puntos más desfavorables; la excavación pudo hacerse sin necesidad de cajón, y en el fondo de la misma se dispusieron 12 pilotes en el perímetro con hasta $4 \mathrm{~m}$ de profundidad bajo la base de la excavación.

Finalmente, mientras que el estribo izquierdo fue cimentado en roca, con una excavación de $7 \mathrm{~m}$, el derecho se situaba en una zona con arena y grava que exigió una excavación de $14,75 \mathrm{~m}$ de profundidad y un conjunto de pilotes de $5,50 \mathrm{~m}$ (fig. 4).

\footnotetext{
2 En ocasiones también se construían viaductos de fábrica, pero en los años 1870 -1880 experimentaban un notable retroceso frente al auge de los puentes de celosía, más económicos y con una ejecución más rápida.
} 


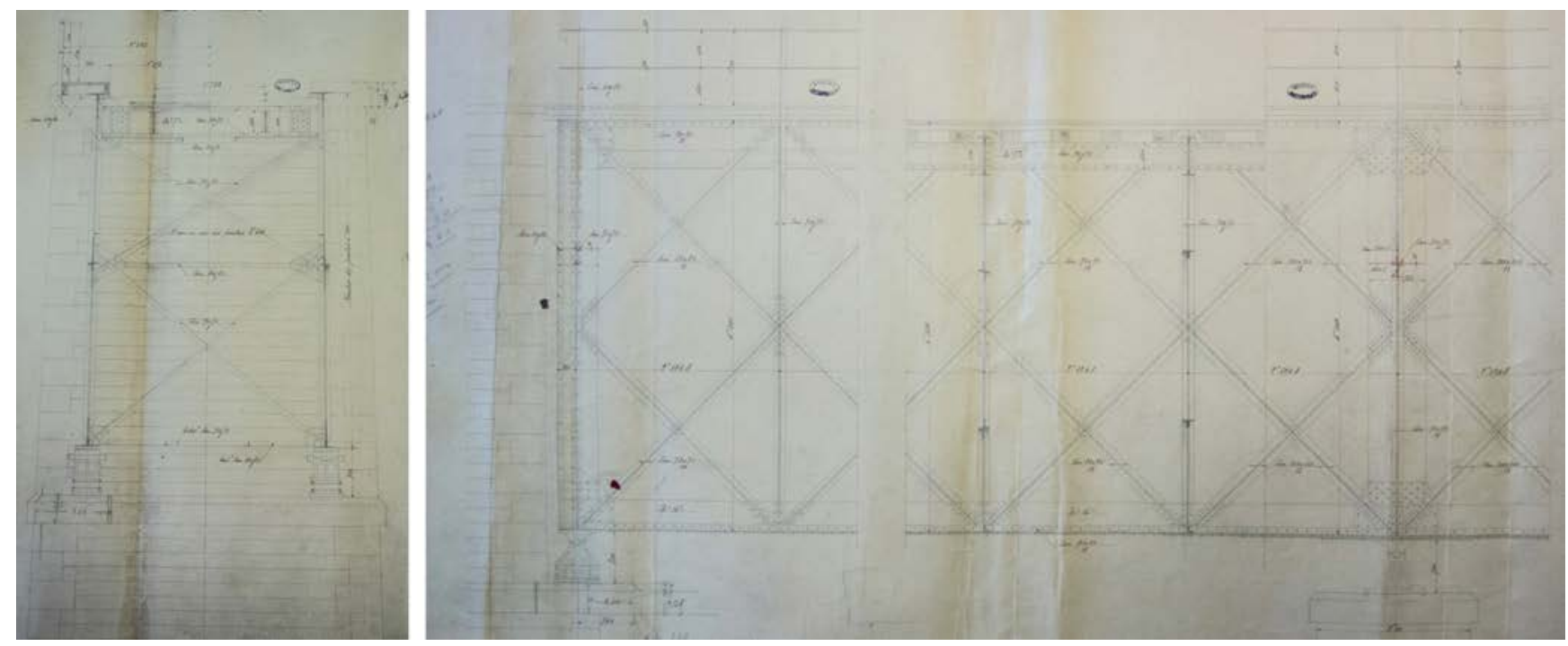

Figura 3. Sección y alzado de una de las vigas (imagen: ANMT).

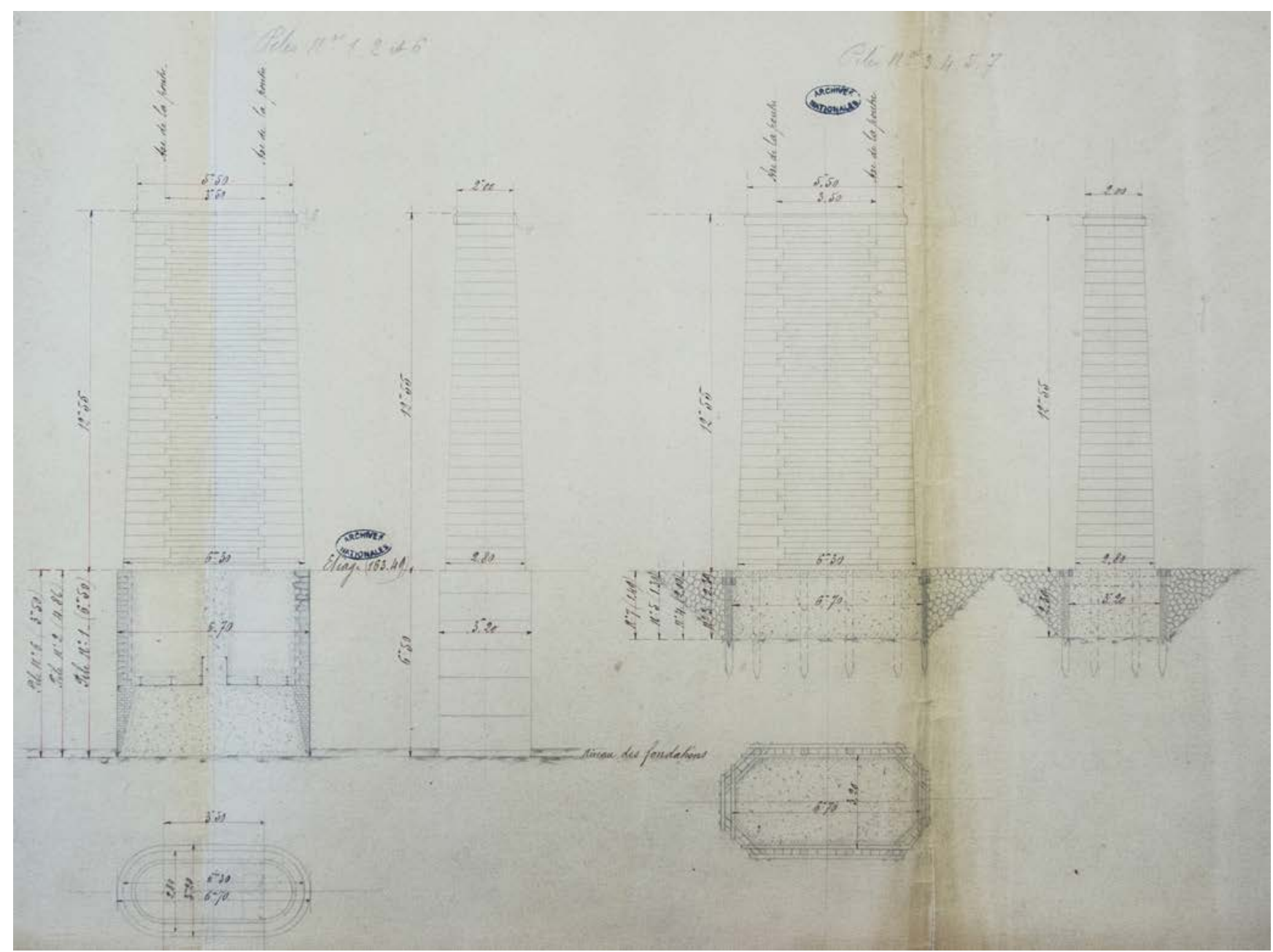

Figura 4. Alzado de las pilas con las dos soluciones: cajón y pilotes (imagen: ANMT).

En cuanto a las cotas (tabla 1), el valor que condicionaba la altura del puente era el de la crecida de 1876 , que llegaba a la cota 174,78 m. Se decidió dejar un resguardo de $2 \mathrm{~m}$, y así la cota inferior de las celosías se estableció en 176,78 m. Si comparamos estos valores con los proyectados para el citado puente coetáneo de Mérida, también en el Guadiana la máxima crecida sobrevino en 1876 , pero en esa ocasión el resguardo proyectado fue de 1,20 m. Cabe recordar que en 1876 tuvo lugar la mayor crecida conocida del río Tajo (9). 

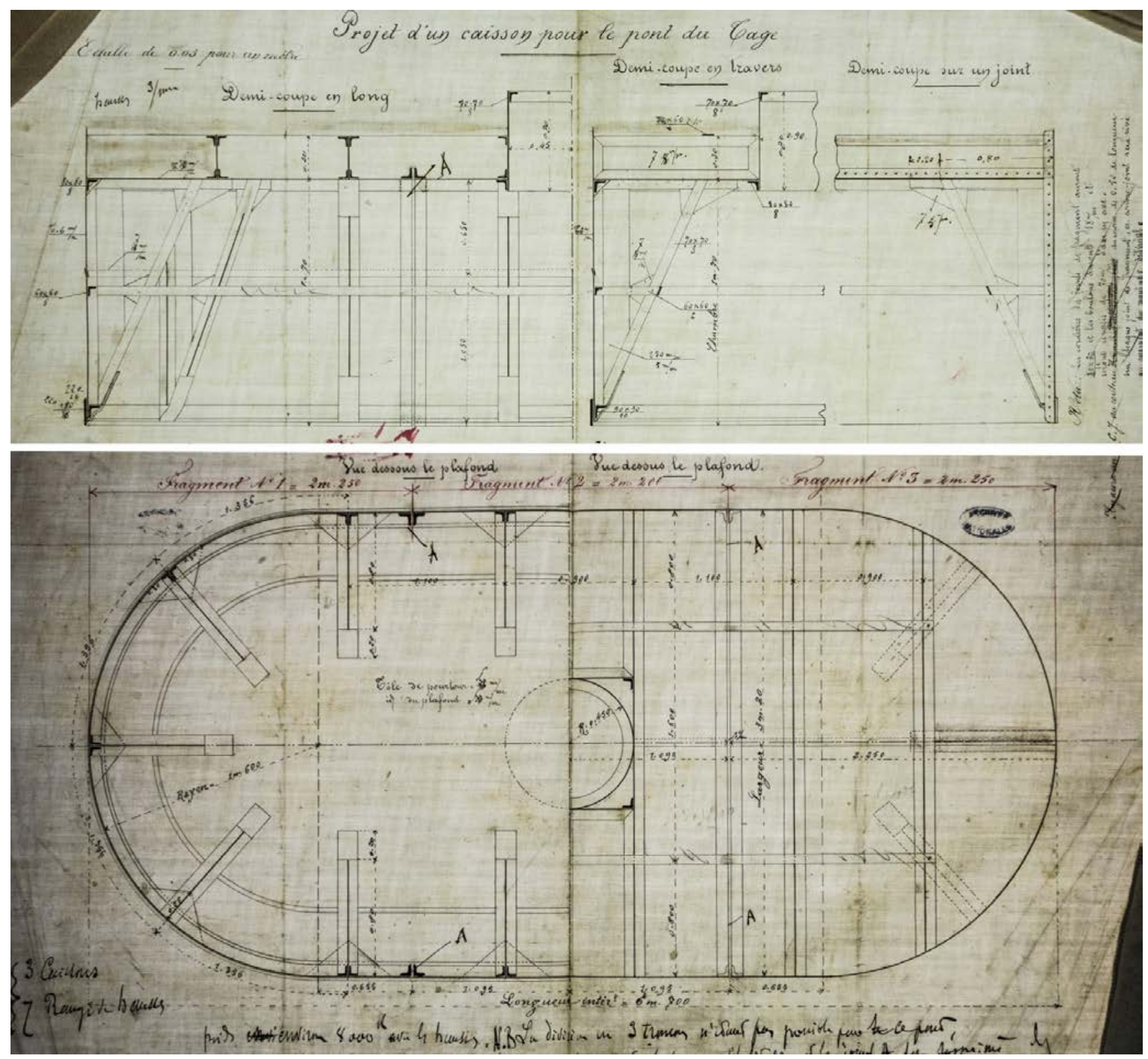

Figura 5. Secciones y planta del cajón para la cimentación mediante aire comprimido (imagen: ANMT).

\subsection{Otros datos de los planos}

El análisis de los planos existentes en el ANMT y en el AHF permite extraer otros datos relevantes.

\section{Planos del ANMT}

Este conjunto de planos, provenientes de los Établissement Eiffel, nos muestran cómo se trabajaba en la empresa de forma interna. Así, había planos de consumo propio, como el correspondiente al cajón de aire comprimido, que se realizaban para ser ejecutados en el propio taller y que no eran delineados posteriormente con especial esmero. Existían también planos para el exterior, como un alzado titulado Chemin de fer de Cacèrès a Malpartida et a la frontière portugaise. Pont sur le Tage; este documento, firmado en Levallois-Perret el 27 de septiembre de 1879, fue impreso por Broise \&
Courtier, imprenta parisina que reproducía los planos de los proyectos de Eiffel $^{3}$. Por último existían distintos planos de construcción, documentos delineados de gran tamaño sin firma pero con fecha que describen el puente con todo detalle, incluso pieza por pieza; sobre estos últimos aparecen numerosas anotaciones a lápiz, correcciones a mano alzada e incluso pequeños cálculos (fig. 7). Y frente a la costumbre de los planos definitivos de proyecto, que suelen firmarse el mismo día, encontramos que cada plano lleva una fecha distinta -no todos la incluían-, permitiéndonos deducir la secuencia del trabajo (tabla 2).

Otro dato relevante es que la casa Eiffel propuso un diseño del puente previo al definitivo, un Avant Projet de soumission (fig. 8). El plano conservado -un plano de trabajo no fechado- permite observar una estructura compuesta por dos partes diferenciadas que salvaba en conjunto una dis-

${ }_{3}$ La referencia del plano es 4419.12.79, y en el mismo se indica la dirección del impresor: 43 R. de Dunkerke, Paris. 

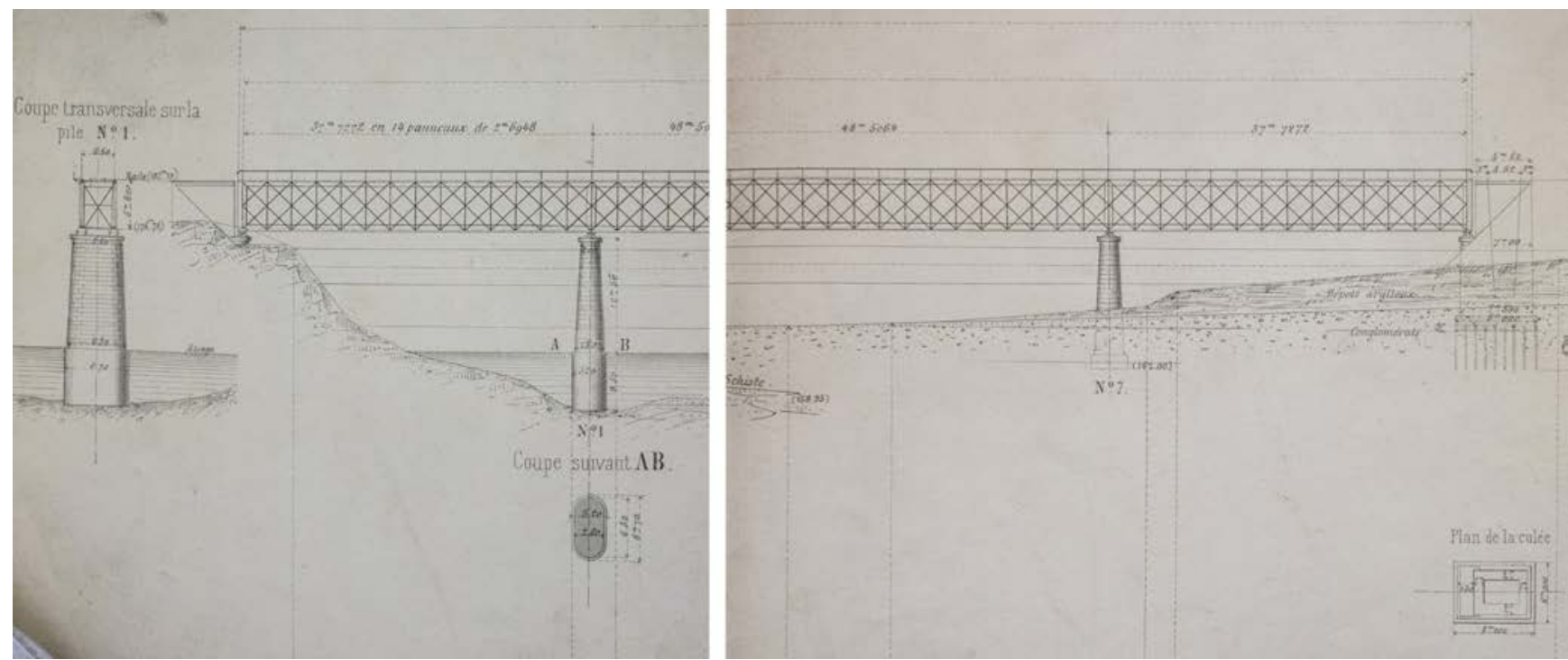

Figura 6. Detalle de los estribos; se observa asimismo la sección de las pilas (imagen: ANMT).

Tabla 1. Cotas reseñadas en el plano. La cimentación se situaba a $156,90 \mathrm{~m}$.

\begin{tabular}{|l|l|}
\hline \multicolumn{1}{|c|}{ Nivel } & \multicolumn{1}{c|}{ Cota } \\
\hline Estiaje (arranque de las pilas) & $163,50 \mathrm{~m}$ \\
\hline Aguas ordinarias & $166,00 \mathrm{~m}$ \\
\hline Aguas altas ordinarias & $170,78 \mathrm{~m}$ \\
\hline Crecida 1876 & $174,78 \mathrm{~m}$ \\
\hline Nivel inferior de la estructura metálica & $176,78 \mathrm{~m}$ \\
\hline Rasante de la explanación & $181,70 \mathrm{~m}$ \\
\hline
\end{tabular}

tancia similar a la del puente definitivo (366 m). La primera parte consistía en un puente de $298 \mathrm{~m}$ compuesto por 6 vanos; las medidas de los extremos era $45,82 \mathrm{~m}$ y la de los cuatro centrales $51,21 \mathrm{~m}$. Las vigas tenían la misma tipología
Tabla 2. Planos que incluían fechas, y secuencia de las mismas.

\begin{tabular}{|l|c|}
\hline \multicolumn{1}{|c|}{ Plano } & Fecha \\
\hline Alzado del puente & 25 septiembre 1879 \\
\hline Detalles de las vigas & 20-21 octubre 1879 \\
\hline Diagrama de momentos flectores y cargas & 17 diciembre 1879 \\
\hline
\end{tabular}

que las definitivas, además de las mismas dimensiones en la sección: 3,50 m x 5,40 m. La segunda parte de la estructura, una suerte de viaducto de aproximación, estaba conformada por una sucesión de cuatro vigas horizontales, de 16,00 m las extremas y de 18,00 m las centrales, apoyadas en pilas metálicas. Las vigas consistían en una celosía más sencilla que las del segmento anteriormente descrito, de 3,50 m de anchura y 2,00 m de altura.
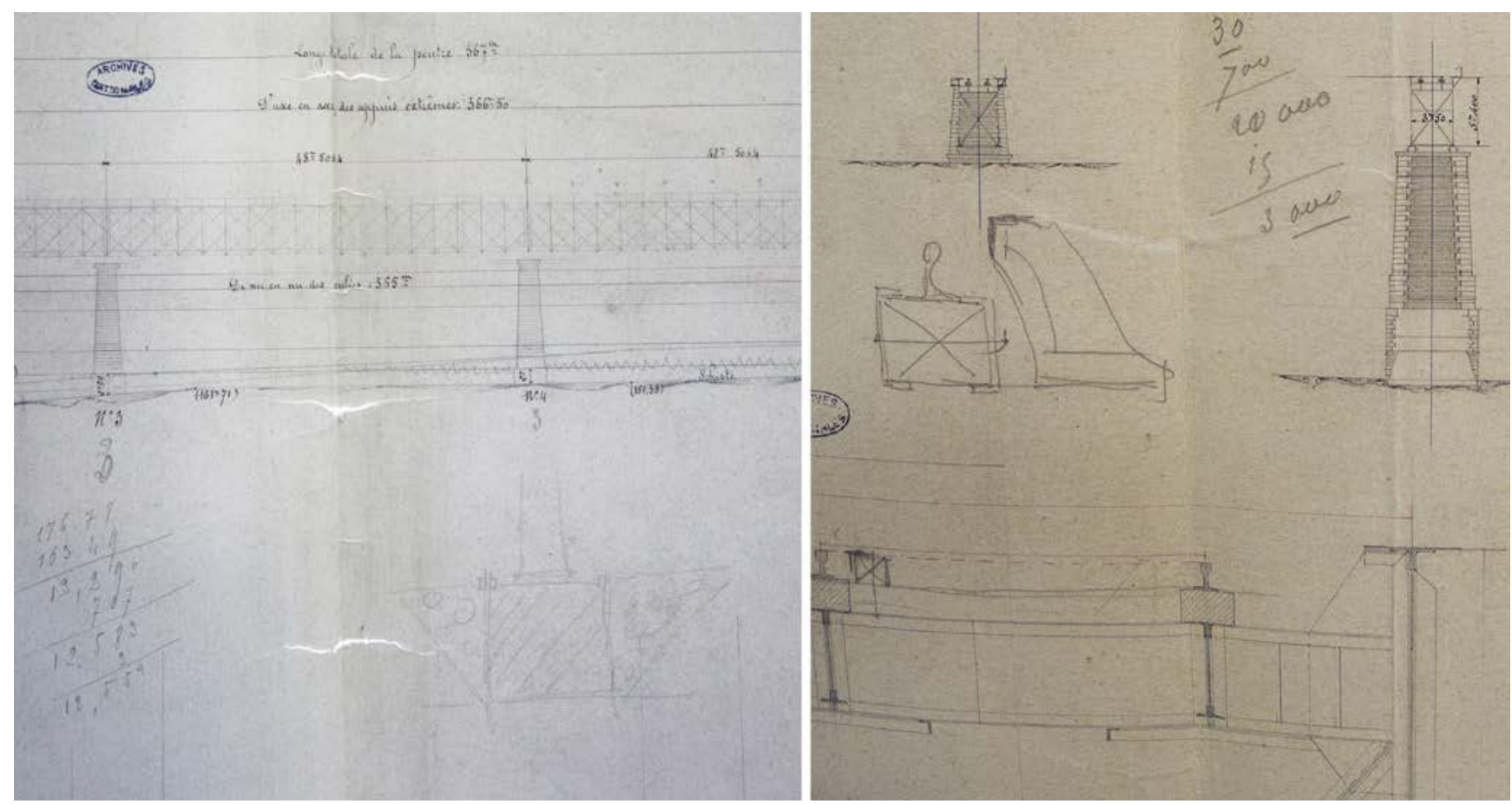

Figura 7. Detalle de anotaciones y dibujos en los planos (imagen: ANMT). 


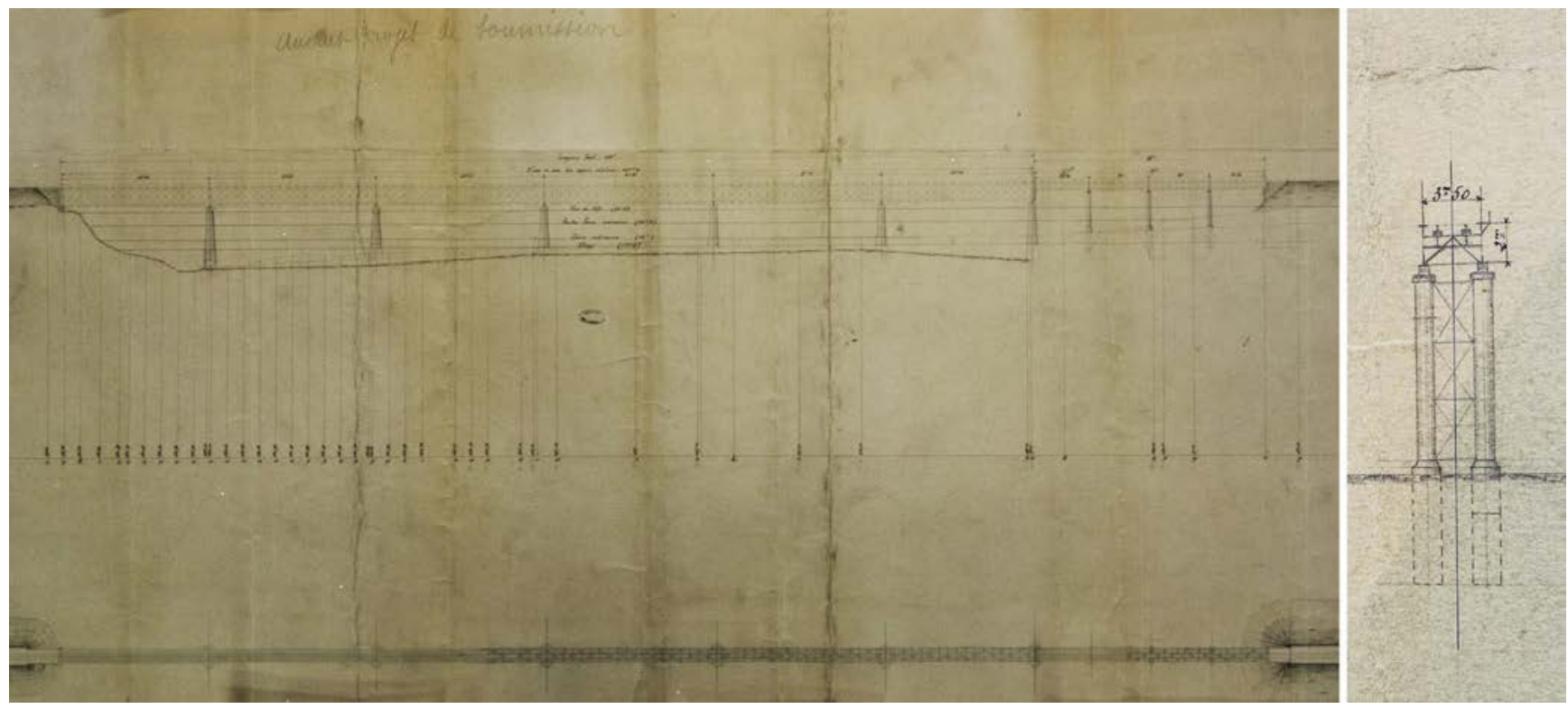

Figura 8. Anteproyecto con la solución de dos partes diferenciadas en la estructura; alzado de las pilas de la mitad derecha (imagen: ANMT).

Un último dato que puede deducirse de los planos es la medida de las traviesas, gracias a la escala del dibujo. Consistían en paralelepípedos de $15 \mathrm{~cm}$ de alto, $25 \mathrm{~cm}$ de ancho y $250 \mathrm{~cm}$ de largo. La separación, de eje a eje, era de unos $67 \mathrm{~cm}$.

\section{Planos del AHF}

Los planos existentes en el AHF de Madrid relativos al puente (10) son copias de algunos de los existentes en Roubaix, reproduciendo la misma escala y conteniendo la misma información. El conjunto es pequeño, e incluye alzado, sección transversal y longitudinal, arriostramientos y apoyos, y diagramas de momentos de flexión y esfuerzo cortante, y carecen de fecha y firma. Su aspecto es más vistoso y claro, pues el dibujo se realizó con distintos colores, frente a los del ANMT simplemente delineados con un color de tinta.

Existe también una copia similar a la del plano de Broise \& Courtier que representa el alzado (11). Fechado el 30 de diciembre de 1879 , señala que fue "aprobado con prescripciones por Real Orden de 26 de mayo de 1880”. El plano incluye las firmas del barón de Covadonga, director general de Obras Públicas; Antonio Martín, ingeniero de construcción; Bonifacio Espinal Ibargoitia, ingeniero de caminos jefe de la compañía; y Segismundo Moret, administrador delegado, y gran impulsor del ferrocarril hacia Cáceres.

\subsection{El proceso de construcción}

La construcción del puente se inició a primeros de 1880. Las pilas y los estribos fueron construidas in situ, mientras que la parte metálica fue construida y montada conforme al sistema productivo usual de la casa Eiffel, que no sólo diseñaba estructuras sino que también producía y cortaba las piezas metálicas de las mismas en sus talleres. El proceso era el siguiente: primero se diseñaba la estructura y cada una de las piezas que la componían, a continuación se producían dichas piezas metálicas en sus instalaciones, y finalmente se enviaban al lugar de la obra, realizándose allí el montaje. Algunos de los ensamblados se realizaban también en las instalaciones de la empresa, como el cajón de aire comprimido que fue trasladado en tres piezas premontadas.
Los talleres Eiffel estaban situados en el 42 de la rue Fouquet de Levallois-Perret, municipio periférico de París que destacaba por su actividad industrial. Las instalaciones ocupaban $20.000 \mathrm{~m}^{2}$, y se describían en Le Petit Journal como un lugar con actividad febril, donde los pedidos se ejecutaban con una exactitud milimétrica, convirtiendo las más diversas estructuras metálicas concebibles en un conjunto detallado de piezas desmontadas que posteriormente se podrían ensamblar con gran rapidez y facilidad en el lugar de destino. Las piezas se producían numeradas y cortadas a medida, y se adaptaban posteriormente como las piezas de un reloj (12), o como las de un mueble sueco. Este sistema de producción permitiría posteriormente erigir la torre Eiffel -7.300 toneladas de material, 18.038 piezas- en apenas 2 años.

El montaje de la estructura metálica, una gran viga continua -pese a estar subdividida en tramos-, se realizaba en las inmediaciones de uno de los estribos, y posteriormente se lanzaba sobre las pilas mediante un proceso novedoso en la época, y que constituyó una de las aportaciones de los Établissement al arte de construir. En un texto escrito por el propio Eiffel (13) se reproduce a modo de apéndice la publicación Les grandes usines de Turgan que describe cómo el ingeniero francés desarrolló unos rodillos múltiples con capacidad de giro sobre su eje horizontal que se situaban sobre las pilas, y que permitían el rodar de las vigas sin que en ningún momento se generasen momentos volcadores sobre las citadas pilas. Estos rodillos permitieron la construcción por el procedimiento del lanzado de obras cuya longitud excedía las posibilidades de dicho procedimiento hasta la fecha. Fueron empleados por primera vez en el puente de la Sioule en 1869 y alcanzó su máximo hito en el puente de Tardes, con 80 $\mathrm{m}$ de altura y cuya luz central alcanzaba los $104 \mathrm{~m}$. Este mismo procedimiento sería asimismo empleado para construir el puente del Tajo -que aparece reproducido en una imagen (fig. 9)- o el de Viana en Portugal.

Apenas hay datos sobre las personas implicadas en los trabajos, más allá de los ingenieros españoles que firman uno de los planos del AHF. En la publicación francesa Annales industrielles se lee que "la línea de Madrid a Cáceres y Portugal ha sido construida por M. Ravel y dirigida por los ingenieros 


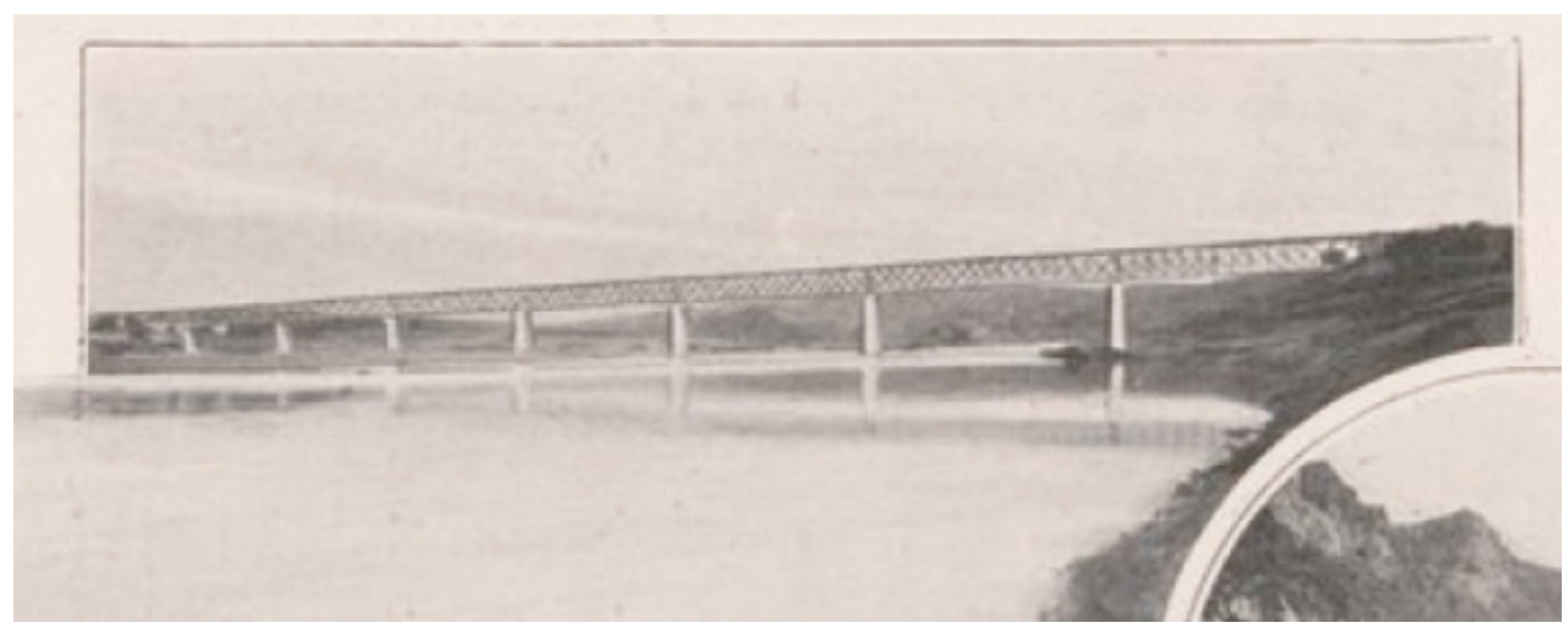

Figura 9. Reproducción del puente del Tajo en la publicación del propio Eiffel.

Vauvilliers y Huguet" (14); posiblemente este último fuera Auguste-Adrien Huguet, director de la Compañía de Ferrocarriles de Madrid a Cáceres y Portugal. Asimismo, en el Anuario de la Escuela Central (15) se indica que el ingeniero Viguié, promoción de 1885, fue inspector de ferrocarriles de la Madrid-Cáceres-Portugal; si bien no pudo -atendiendo a la fecha- participar en la construcción del puente, sí confirma la fuerte implicación de ingenieros franceses en esta línea ferroviaria. No en vano, muchas de las decisiones relevantes de la compañía se tomaban en París (16).

\subsection{Construcción de la línea del telégrafo y posterior desguace}

Inaugurado en octubre de 1881, el puente de Eiffel permaneció en servicio hasta 1932; previamente había sido aprovechado hacia 1926 por la compañía Telefónica para tender la línea del telégrafo (17). A tal fin se colocó en cada pila del puente un apoyo -consistente en un larguero de madera de 1,10 m- para la colocación de los aisladores; la instalación era provisional, puesto que estaba previsto que el tendido definitivo se realizase por el puente de la carretera, que estaba en construcción en ese momento.

El 24 de septiembre de 1925 se promulgó la Instrucción oficial para la redacción de proyectos de tramos metálicos, que definía las nuevas exigencias que debían tener los puentes ferroviarios, debido al aumento del peso del material móvil. La mayor parte de puentes metálicos fueron sustituidos al incumplir los nuevos parámetros resistentes. Ante este hecho, cabían dos opciones: reemplazar exclusivamente la parte metálica de la estructura o construir un puente enteramente nuevo. Un ejemplo de la primera opción es el ya citado puente del Guadiana de Mérida, donde la estructura original de hierro fue sustituida por otra formalmente similar, pero de acero. La segunda opción se llevó a cabo, por ejemplo, en los puentes de hierro de Zarza de Alange y Aljucén, que fueron sustituidos por otros de hormigón y fábrica (18).

En el caso del puente de Eiffel, se decidió erigir un puente nuevo de hormigón en masa y mampostería a partir de un proyecto de Fernando del Pino, ingeniero de la Compañía nacional de los ferrocarriles del oeste de España, explota- dora en ese momento de la línea. Él mismo justificaba que construir la nueva estructura resultaba la opción más económica al limitarse su presupuesto a 1.300.00o pesetas, si bien no detallaba cifras de las otras opciones (19). En el texto se obvia el interés patrimonial del puente de Eiffel: "se eligió (...) la obra de fábrica como más económica y huelga decir que como más conveniente, también desde los demás puntos de vista”. Años después, con motivo de la inundación de la estructura causada por la construcción de la presa de Alcántara, escribiría: "siendo ingeniero jefe (...) no era mi cometido, ni para ello tenía tiempo (...) pero en aquella ocasión me encapriché con el puente, y como era el jefe... pues lo hice, a ratos perdidos. Mala cosa, porque le tomé cariño" (20). Un texto donde sobrevuela, quizá, su interés en proyectar un puente nuevo frente a la opción de adaptar el existente.

Tras la entrada en servicio del nuevo puente hacia 1932, quedaba pendiente decidir el destino del obsoleto. Existiendo distintas alternativas, como el abandono o la reutilización para otro uso, se optó por demolerlo. Debemos señalar que el proyecto del nuevo puente sugería ya tomar esta decisión, pero sin presupuestarla.

Así, se redactó un Proyecto de Desguace del tramo metálico y demolición de las pilas de fábrica del puente sobre el río Tajo (21), firmado en Madrid el 12 de agosto de 1932 por el ingeniero jefe de vía y obras de la compañía, de apellido Delgado (acaso Manuel Delgado Delgado), que justificaba la decisión de eliminar el puente de Eiffel: ambas estructuras tenían las pilas alternadas, por lo que mantener en pie los apoyos del puente antiguo resultaba contraproducente desde el punto de vista hidráulico. Al ser las pilas obstáculos para el fluir natural de las aguas, se pensaba que la adición de un nuevo conjunto de pilas en un espacio físico cercano al conjunto del puente antiguo podía dificultar el desagüe del río. Debido a ello era preciso desmontar en primer lugar el tramo metálico del puente de Eiffel y a continuación demoler sus pilas. La Memoria del proyecto señalaba: "no debemos dejar de consignar la urgencia (...) una vez construido el nuevo puente, ya que cuanto antes desaparezcan los obstáculos que en el cauce supone las pilas viejas para las próximas avenidas del río, antes quedará éste en las debidas condiciones para su desagüe normal”. 
El desmontaje de la viga metálica, cuyo peso se estimaba en 700 toneladas, se realizó por partes desde el nuevo puente construido con la ayuda de una grúa. En relación a las pilas, causantes en teoría de la demolición, nunca llegaron a demolerse, tal y como atestiguan distintas fotografías posteriores (fig. 10). Quizá -pensamos- se decidiera eliminar la parte metálica porque se le podía sacar un beneficio económico vendiéndola como chatarra, y la cuestión hidráulica de la acumulación de pilas resultara una excusa para lograr tal fin.

Del mismo modo, señalamos el error de Del Pino al situar su puente tan cercano al de hierro (apenas 6,16 m), pues si ambas estructuras se hubieran separado más, quizá la de Eiffel hubiera permanecido en pie. Asimismo, también podría haber situado las pilas de su puente en una ubicación compatible con las del puente antiguo.

\section{EL IMPACTO DEL PUENTE}

El puente del Tajo fue uno de las principales estructuras construidas por Eiffel. Un texto de Cléry de 1911 (22) lo consideraba uno de los once grandes puentes del ingeniero (tabla 3), y el propio Eiffel, en su discurso de aceptación de la Legión de Honor en 1889 , lo citaba como una de sus obras fundamenta-

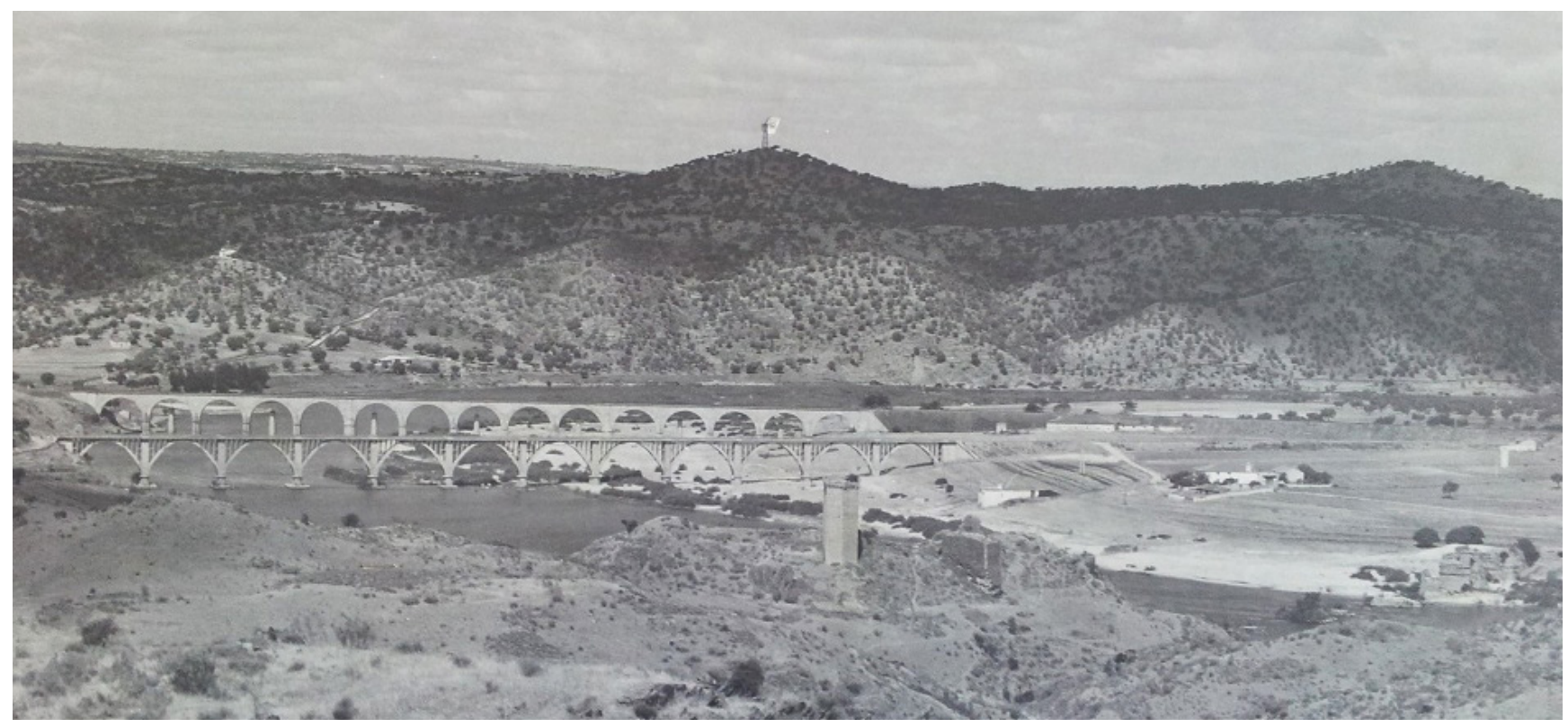

Figura 10. Vista del vado de Alconétar hacia 1950. Se observan los puentes carretero, ferroviario y las pilas del de Eiffel en los vanos no consecutivos de este último.

Tabla 3. Principales puentes de Eiffel según Cléry.

\begin{tabular}{|c|c|c|c|c|c|c|c|}
\hline Puente & Año & Rio & País & Tipología & Vanos & $\begin{array}{l}\text { Otros ingenieros } \\
\text { implicados }\end{array}$ & $\begin{array}{l}\text { Estado } \\
\text { actual }\end{array}$ \\
\hline Maria Pia & $1876-1877$ & Duero & Portugal & $\begin{array}{l}\text { Arco y } \\
\text { celosía }\end{array}$ & $\begin{array}{c}352,875 \mathrm{~m} \\
(\operatorname{arco~de~} 160 \mathrm{~m})\end{array}$ & $\begin{array}{l}\text { T. Seyrig, M. } \\
\text { Augevére; P. I. } \\
\text { Lopes }\end{array}$ & Cerrado al uso \\
\hline Puente de Viana & 1878 & Lima & Portugal & Celosía & $562 \mathrm{~m}$ en 10 vanos & $\begin{array}{l}\text { T. Seyrig, J. Matos; } \\
\text { B. Vieira }\end{array}$ & En servicio \\
\hline Cubzac & $1879-1883$ & Dordoña & Francia & Celosía & $\begin{array}{c}57,60 \mathrm{~m}-6 \times 72,80 \mathrm{~m}- \\
57,60 \mathrm{~m} \\
\end{array}$ & $\begin{array}{l}\text { Alard, Descombes, } \\
\text { Nouguier, Poirier }\end{array}$ & Reconstruido \\
\hline Puente del Tajo & 1881 & Tajo & España & Celosía & $\begin{array}{c}37,72 \mathrm{~m}-6 \times 48,51 \mathrm{~m}- \\
37,72 \mathrm{~m} \\
\end{array}$ & & Destruido \\
\hline $\begin{array}{c}\text { Messageries } \\
\text { maritimes } \\
\text { (o Pont Mong) }\end{array}$ & 1882 & Rach Bên Nghé & Vietnam & $\begin{array}{l}\text { Arco } \\
\text { rebajado }\end{array}$ & $80 \mathrm{~m}$ & & En servicio \\
\hline Tân An & $1882 ?$ & Vam Co Tay & Vietnam & Celosía & $\begin{array}{c}7 \text { vanos, el mayor de } \\
80 \mathrm{~m}\end{array}$ & & Destruido \\
\hline Bên Lúc & $1882 ?$ & Delta del Mekong & Vietnam & Celosía & $516 \mathrm{~m}$ en 15 vanos & & Destruido \\
\hline Szeged & 1883 & Tisa & Hungría & $\begin{array}{c}\text { Arcos } \\
\text { rebajados }\end{array}$ & $\begin{array}{c}110,30 \mathrm{~m}-97,00 \mathrm{~m}- \\
96,00 \mathrm{~m}-66,00 \mathrm{~m}\end{array}$ & J. Feketeházy & Destruido \\
\hline Tardes & 1884 & Tardes & Francia & Celosía & $\begin{array}{c}73.20 \mathrm{~m}-104.55 \mathrm{~m}- \\
73.20 \mathrm{~m}\end{array}$ & $\begin{array}{l}\text { Guillaume; } \\
\text { Daigremont }\end{array}$ & En servicio \\
\hline Garabit & 1884-1886 & Truyère & Francia & $\begin{array}{l}\text { Arco y } \\
\text { celosía }\end{array}$ & $\begin{array}{c}565,00 \mathrm{~m} \\
\text { (arco de } 165 \mathrm{~m} \text { ) }\end{array}$ & $\begin{array}{l}\text { L. Boyer, M. } \\
\text { Koechlin }\end{array}$ & En servicio \\
\hline $\begin{array}{l}\text { Conflans-Sainte- } \\
\text { Honrine }\end{array}$ & 1892 & Oise & Francia & Celosía & Principal: $100 \mathrm{~m}$ & & Sustituido \\
\hline
\end{tabular}




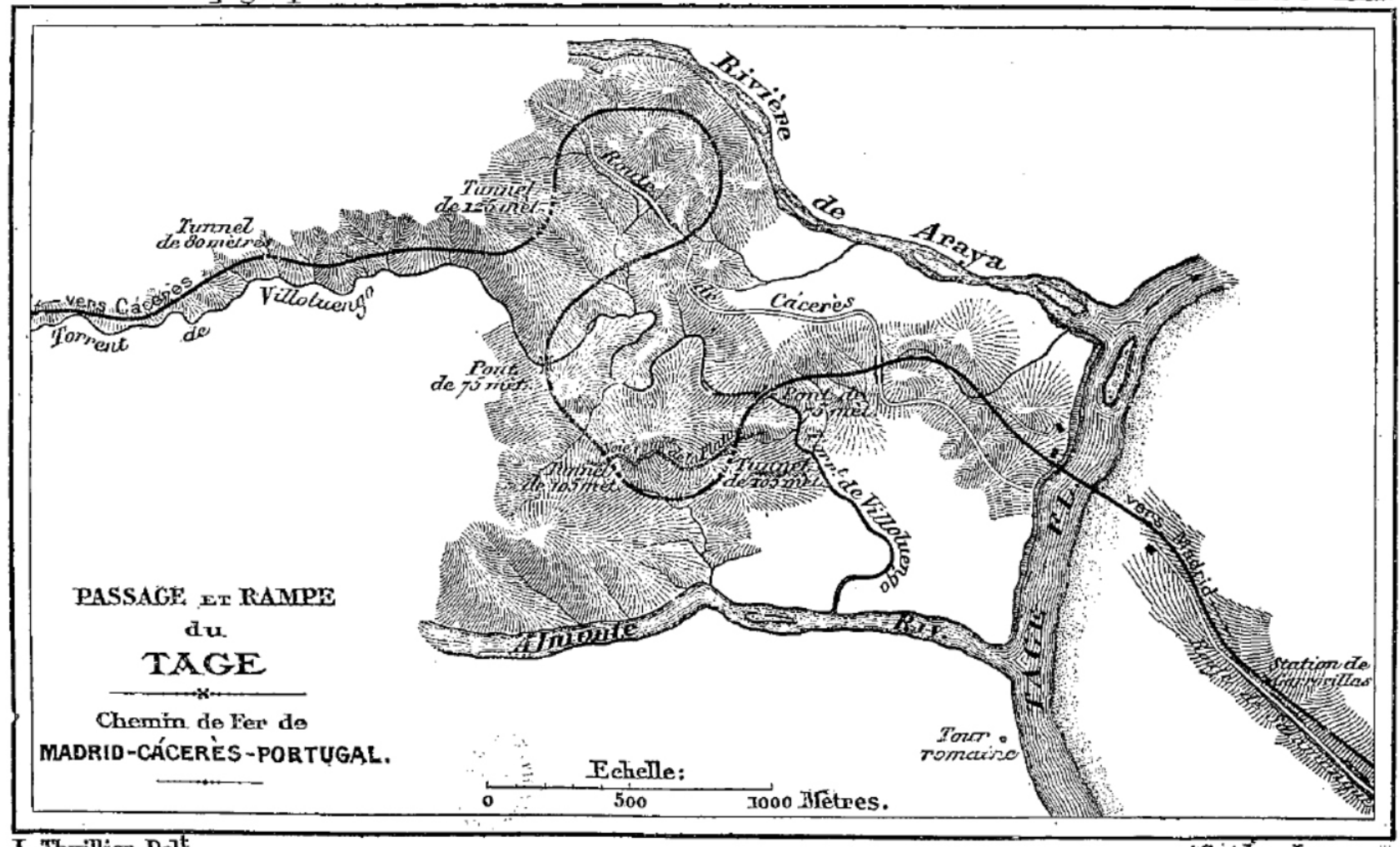

L.Thrillier, Det:

Guides Joanne.

Figura 11. La zona de Alconétar, del libro de Lavigne. Se observan distintos puentes y túneles, además del puente de Eiffel.

les, junto a los viaductos en los Andes, los puentes de Oporto, de Szagadine, el de Rumanía, de Tonkin y los de la antigua Cochinchina, y la estación ferroviaria de Pest (23).

Otros textos recogen también alusiones a la estructura, como la guía de viajes de Germond de Lavigne de 1890 que lo destaca como "un beau pont en fer" (24), reproduciendo además un mapa del vado de Alconétar para describir el trazado de la línea férrea en ese punto (fig. 11), que consideraba una de las más complejas de la Península junto a otras como las rampas de Orduña y Barcena, el trazado de La Granja y los pasos a Asturias y Galicia (25). En la Revista de Obras Públicas (26), se alababa su gran ligereza en un texto que ya sentenciaba la necesidad de sustituirlo de acuerdo con la Instrucción señalada anteriormente. Publicaciones más recientes, como la monografía de Eiffel escrita por Lemoine lo destaca como un puente preeminente dentro de la obra eiffeliana (27).

Pero acaso la prueba más nítida de la relevancia de la estructura fuera el hecho de que el ingeniero francés fue distinguido, tras la construcción del mismo, con el título de Comandante de la Orden de Isabel la Católica en España (28).

\section{CONCLUSIONES}

El análisis de los distintos datos relativos al puente de Eiffel permite extraer una serie de conclusiones.

En primer lugar, se resalta la importancia del puente del Tajo construido por Eiffel. Además de la natural notabilidad que tiene de por sí un puente de hierro diseñado y construido por uno de los ingenieros más célebres de la historia -quizá el más popular de todos-, en la estructura que nos ocupa están presentes cuestiones como el sistema de producción, el método constructivo de lanzado de la viga, el cajón de aire comprimido para la cimentación o la propia tipología estructural junto con los diagramas de fuerzas y momentos, que en ese tiempo eran punteros en cuanto a tecnología en el arte de la construcción de puentes. Cabe asimismo resaltar la ligereza de una estructura de $367 \mathrm{~m}$ con una luz máxima de 48,51 $\mathrm{m}$, cuyo peso de 700 toneladas arroja un peso lineal de 1,91 toneladas $/ \mathrm{m}$.

En segundo lugar, se ha aportado algo más de luz al modo en que se trabajaba en la propia compañía Eiffel. El análisis de los documentos de Roubaix y Madrid han permitido observar cómo se elaboraba al inicio una propuesta definida por un alzado, que sería posteriormente revisada por la definitiva; después se trazaban una serie de planos de consumo interno revisados por los ingenieros de los propios Établissement, que definían cada una de las piezas a producir; finalmente, se enviaban al cliente los planos ya terminados y éste les daba también el visto bueno.

En tercer lugar, debe lamentarse la decisión de demoler el puente frente a la posibilidad de reemplazar la estructura de hierro por otra de similar tipología pero de acero, o de construir un puente nuevo manteniendo en pie el antiguo. El motivo para demoler la estructura fue absurdo, porque se hizo como paso previo y necesario para demoler las pilas, y al final éstas permanecieron en pie -de hecho permanecen aún en pie, sumergidas-. Y el lamento se convierte en incomprensión, pues se trataba de eliminar una obra de un ingeniero ya célebre a nivel mundial, cuya torre parisina había sido ya indultada. El puente del Tajo, además, era rele- 
vante fuera de las fronteras españolas tal y como atestiguan los textos franceses de 1910-1920. Hoy día, en fin, se tiende a identificar cualquier estructura roblonada con una obra eiffeliana, a fin de añadir a la misma una pátina distintiva y un incontestable valor patrimonial (29). Pues bien, hace 80 años se decidió demoler un auténtico puente Eiffel sin problema alguno.

La demolición hermana el puente de Eiffel con el puente de Alconétar, arruinado en el marco de la Reconquista. Ambas estructuras, que mostraban el brío y el ingenio de los técnicos de sus respectivos tiempos históricos, perdieron su función de unir dos orillas por la acción del hombre.

Un pensamiento derivado de la decisión de demoler el puente es la desprotección de las infraestructuras históricas situadas en el medio rural. En las ciudades abundan los casos de puentes obsoletos que siguen jugando un papel en la red de transportes, merced a peatonalizaciones o rehabilitaciones; sin embargo las construcciones situadas en espacios escasamente poblados son abandonadas a su suerte, demolidas o vandalizadas. Sí es verdad que en tiempos recientes, en España se tiende a reconvertir las líneas férreas abandonadas o desmanteladas en vías verdes (30), recuperando así viaductos obsoletos y degradados.
Por último, cabe preguntarse qué hubiera ocurrido en los años 1960 si el puente hubiera seguido en pie. La inundación del lugar donde se asentaba en 1969 habría planteado el debate del traslado de la estructura -como se hizo con el puente de Alconétar- o la pérdida del mismo bajo las aguas. Esta reflexión deja en el aire otras cuestiones: ¿tendría más valor un puente romano que uno de Eiffel? ¿Qué elementos patrimoniales deben ser salvaguardados en caso de que estén amenazados por una eventual inundación? ¿Está regulado cómo actuar ante estas situaciones? Si bien hoy día parece poco probable que se construyan grandes embalses en países como los europeos, existen otros con ambiciosos planes de construcción de presas donde estas cuestiones pueden ser aún relevantes.

\section{AGRADECIMIENTOS}

La investigación ha sido financiada por el proyecto «La patrimonialización de un territorio: conformación de paisajes culturales entre el Tajo y el Guadiana en Extremadura (HAR 2013-41961-P)», financiado por el Ministerio de Economía y Competitividad (Gobierno de España). El autor agradece la ayuda de Vicente Plasencia Lozano en el tratamiento de las imágenes, así como a J.M. Mellado por la fotografía y a Hadrián Arias por sus observaciones en versiones previas del manuscrito.

\section{REFERENCIAS}

(1) Plasencia-Lozano, P. (2017, 2-6 Octubre). El proyecto no construido del ferrocarril entre Talavera de la Reina y Cáceres por Trujillo de Eusebio Page, y la modificación de Ángel Arribas. En X Congreso Nacional y II Congreso Internacional Hispanoamericano de Historia de la Construcción. San Sebastián: Instituto Juan de Herrera.

(2) Lozano Bartolozzi, M. M., \& Plasencia Plasencia, V. (1986, 16-20 de junio). Los puentes del Tajo en Alconetar. En Los caminos y el arte: VI CEHA. Santiago de Compostela: Servicio de publicaciones USC.

(3) Plasencia-Lozano, P. (2012). Alconétar, paisaje cultural de la ingeniería. Una propuesta de ordenación territorial. En Lozano Bartolozzi, M. M. \& Méndez Hernán, V. (Eds.), Paisajes modelados por el agua: entre el arte y la ingeniería (pp. 187-205). Cáceres: Editora Regional de Extremadura.

(4) Plasencia-Lozano, P. (2017, 2-6 Octubre). El proyecto no construido del ferrocarril entre Talavera de la Reina y Cáceres por Trujillo de Eusebio Page, y la modificación de Ángel Arribas. En X Congreso Nacional y II Congreso Internacional Hispanoamericano de Historia de la Construcción. San Sebastián: Instituto Juan de Herrera.

(5) Plasencia-Lozano, P. (2016). An analysis of Merida's iron railway bridge: an example of a Linville truss bridge in Spain. Construction History, 31(1): 161-172.

(6) Gaceta de Madrid. http://www.boe.es/datos/pdfs/BOE//1877/187/Aooo41-00042.pdf

(7) Heredia Campos, M. del C. (2009). La elegancia de lo útil. El legado de hierro de Eiffel en España. Revista Del Ministerio de Fomento, (590): 62-69.

(8) ANMT 152 AQ 305.

(9) Uribelarrea, D., Díez Herrero, A., \& Benito, G. (2004). Actividad antrópica, crecidas y dinámica fluvial en el sistema Jarama-Tajo. En Itinerarios geomorfológicos por Castilla-La Mancha (pp. 83-121). Madrid: CSIC.

(10) AHF M-0005-027.

(11) AHF M-0004-009.

(12) Henry. (1890, November 30). La grande industrie. Le Petit Journal, p. 5. Paris.

(13) Eiffel, G. (1900). Travaux scientifiques executés a la tour de trois cent mètres de 1889 a 190o, pp.230-232. Paris: L. Maretheux.

(14) No Title. (1889, January 27). Annales Industrielles, p. 111.

(15) Barré, R. (Ed.). (1889). Les anciens élèves de L’Ècole Centrale 1832-1888, p.66. Paris: Nouvelle Association Ouvrière.

(16) Silva, Á. (2014). Organizational Innovation in Nineteenth-Century Railway Investment: Peripheral Countries in a Global Economy. Business History Review, 88(04): 709-736. doi: 10.1017/Sooo7680514000737.

(17) AHF I-0269-008.

(18) Plasencia-Lozano, P. (2016). An analysis of Merida's iron railway bridge: an example of a Linville truss bridge in Spain. Construction History, 31(1): 161-172.

(19) Del Pino, F. (1933). Nuevo puente sobre el río Tajo en el kilómetro 299,3 de la línea de Madrid a Valencia de Alcántara, de la Compañía Nacional de los Ferrocarriles del Oeste de España. Revista de Obras Públicas, 2616(1): $103-111$.

(20) Del Pino, F. (1969). Réquiem por un puente. Revista de Obras Públicas, 3051(02): 527-530.

(21) AGA 24/8881.

(22) Cléry, A. (1911, June 15). No Title. L’Aérophile, pp. 265-266. Paris. 
(23) Eiffel, G. "A la Tour Eiffel”, Le Petit Parisien (Paris). 23/04/1889.

(24) De Lavigne, G. (1890). Espagne et Portugal, p. 418. Paris: Hachette.

(25) De Lavigne, G. (1890). Espagne et Portugal, p. XXV. Paris: Hachette.

(26) Mariño Ortega, V. (1926). Puentes sobre el Tajo. Revista de Obras Públicas, 76(2506): 272-276.

(27) Lemoine, B. (2002). Gustave Eiffel. Madrid: Akal.

(28) Eiffel, G. (1900). Travaux scientifiques executés a la tour de trois cent mètres de 1889 a 190o, p. 256. Paris: L. Maretheux.

(29) Hernández Lamas, P., \& Bernabéu Larena, J. (2014). Los paisajes culturales de la ingeniería: tópicos a evitar en la consideración de las obras públicas. Revista de Obras Públicas, 3559(161): 87-98.

(30) Aycart Luengo, C. (2001). Vías verdes "Greenways", to reuse disused mil-wais line for non motorised itineraries, leisure and tourism. Informes de la Construcción, 53(475). doi: 10.3989/ic.2001.v53.i475.653. 